\title{
Scenario Reduction Method based on Output Performance for Condition-Based Maintenance Optimization
}

\author{
Xinbo QIAN*, Qiuhua TANG** \\ *Key Laboratory of Metallurgical Equipment and Control Technology of Ministry of Education, Hubei Key Laboratory of \\ Mechanical Transmission and Manufacturing Engineering, Wuhan University of Science and Technology, Wuhan, China, \\ 430081,E-mail: xinboqian@wust.edu.cn \\ **Department of Industrial Engineering, Wuhan University of Science and Technology, Wuhan, China, 430081, \\ E-mail: tangqiuhua@wust.edu.cn
}

cross $^{\text {ref }}$ http://dx.doi.org/10.5755/j01.mech.23.5.15435

\section{Introduction}

Condition-based maintenance (CBM) is a maintenance program that recommends maintenance decisions based on the information collected through condition monitoring [1]. CBM is one of the important maintenance methodologies for prognostics and health management (PHM) [2]. Since the degradation process for componentlsystem is mainly nonlinear and non-stationary, forecasting of degradations is of uncertainty [3]. Therefore, it is essential to deal with CBM optimization incorporating stochastic prognostics [1]. Degradation forecasting is one of important prognostics for CBM optimization, which includes physicalbased model [4], data-driven model [5, 6] and reliability model combing monitoring data $[1,7]$.

For CBM incorporating with stochastic degradation, the current research work can be divided into two types in accordance of the planning horizon: infinite horizon and finite horizon [8]. CBM for a specified finite horizon can be more realistic than that for infinite horizon. In particular, the degradation possibility distribution can be dynamical and non-stationary [9]. Simulation-based CBM model can be applied to deal with this problem [10]. However, for the simulation-based CBM optimization, the computational complexity can increase in exponent with the number of monitoring conditions and stages. Therefore, it is essential to explore effective methods to reduce the computational complexity for CBM within the finite horizon. In other words, effective discretization methods for forecasting degradations can be considered a way to solve the bottle-neck in reducing computational complexity for $\mathrm{CBM}$ in finite horizon.

Uncertainty also exists in decision making problems for other application areas, as well as for CBM. Many computational methods for solving this kind of problem are based on approximating the original uncertainty distribution by discretization of the possibility model presenting uncertainty with finitely many scenarios [11]. It is natural to try to approximate the original scenarios set with much smaller subset that keeps key characteristics, and this process is called scenario reduction, as shown in Fig. 1. In general, fine discretizations can lead to numerically intractable problems, but coarse discretizations may lead to questionable model by neglecting important scenarios. Thus, scenario reduction has been an important topic in the past decades addressing uncertainty in decision making mainly for power system by stochastic programming [12-14].

The basic idea of scenario reduction is to select a few representative scenarios among a large number of scenarios (Fig. 1), and to assign probability to each selected scenario, so as to span possible output scenarios and capture wide range of outcomes in a way that is useful for decisionmaking [11]. According to the reduction mechanisms, current research of scenario reduction is mainly based on the input performance of uncertain parameters [13-15]. For scenario reduction based on input performance, the distance of scenarios is measured by the probability metric distance, and then the possibilities are redistributed among the selected scenarios by distance given in terms of mass transportation problems, to minimize the probability distance between initial and reduced scenarios. Heitsch and Römisch [15] proposed a kind of fast forward and backward reduction to reduce the computational complexity, applying the discretization of possibility distribution instead of the initial continuous probability distribution. A scenario optimal reduction technique, introduced by Dupačová et al [14], applied the Foret-Mourier distance and duality theory to compute the distance between two probability measures. Another variant of the scenario optimal reduction, introduced by De Oliveira et al. [13], combined with global and local (stage-wise) reduction, was to select a small set of sequences representing the stochastic process well enough.
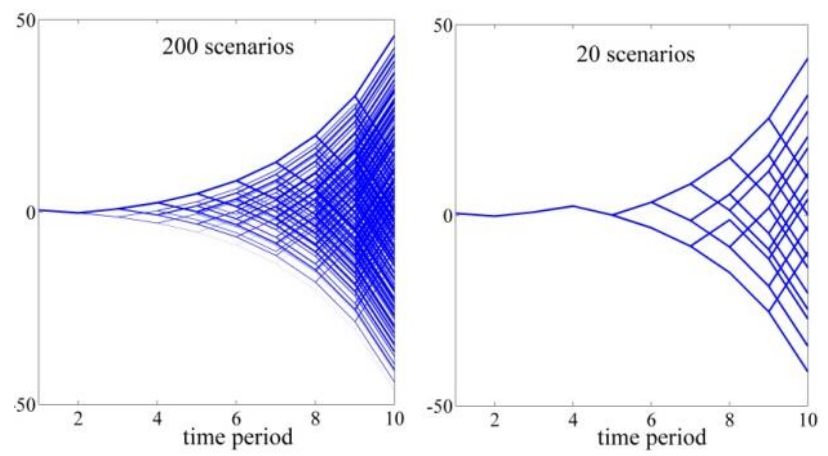

Fig. 1 Initial scenarios (left) and reduced scenarios (right)

These scenario reduction methods based on input performance focus mainly on the scenario parameters, but overlooking where the uncertainties appear in the problem modelling and their impacts on the optimal decision [16]. To deal with this problem, a novel scenario reduction method is proposed mainly based on the CBM model features. In other words, it is a kind of problem-dependent scenario reduction method, instead of isolating the scenario reduction from the problem. 
The paper is organized as follows: Firstly, CBM optimization model and degradation model are introduced in Section 2 and 3, respectively. Secondly, in Section 4, we propose the scenario reduction based on the output-performance for forecasting degradations, aim to increase the computational efficiency of the CBM optimization. A case study based on degradation data from rotating machinery is presented in Section 5. Finally, conclusions and future works are outlined in Section 6.

\section{Control-limit policy for CBM}

For a single-unit system which degrades during the operation, the degradations can be detected by sensors at equally spaced discrete time epochs $k, k=0,1,2 \ldots N T$, with $\Delta t$ being the constant time interval between two consecutive observations. The system is considered to fail whenever its degradation level reaches a given (i.e. not a decision variable) failure threshold [17]. The decision variable is preventive maintenance (PM) threshold $d_{1}^{i}$ based on the monitoring degradation scenario. The expected maintenance cost rate including the costs entailed by corrective maintenance (CM), PM within the planning horizon is written as follows:

$$
Q\left(d_{1}^{i}, Z_{t}^{i}\right)=\sum_{t=1}^{N T}\left(c_{F} I F_{t}^{i}+c_{P} I P_{t}^{i}\right),
$$

where $c_{F}, c_{P}$ are, respectively, CM and PM maintenance cost per time. We define $I F_{t}^{i}$ and $I P_{t}^{i}$ are, respectively, the indicator whether the system should be taken CM action, the indicator whether the system should be taken PM action, at time epoch $t$ for degradation scenario $i$. The control-limit policy for CBM for a single-unit system is as follows [18]:

1) For each scenario $i$, if the degradations $Z_{t}^{i}$ exceeds the failure threshold, CM will be triggered. CM cost will be incurred and $I F_{t}{ }^{i}=1$. Otherwise, $I F_{t}{ }^{i}=0$.

2) PM actions will be triggered if the degradation is above PM threshold but it does not exceed the failure threshold. PM cost will be incurred as a result and $I F_{t}^{i}=1$. Otherwise, $I F_{t}^{i}=0$.

3) No maintenance actions are required if the degradation is below PM threshold. Therefore, no maintenance will be incurred, and both $I F_{t}^{i}=0$ and $I P_{t}^{i}=0$. For the CBM model, we assume that the system is as good as new after each maintenance action. As a result, the degradation of the system after each maintenance is equal to $W_{1}^{i}$, if $I F_{t}^{i}+I P_{t}^{i}>1$. Otherwise, the degradation will follow the forecasting model, i.e., $Z_{t+1}^{i}=W_{t+1}^{i}$. Furthermore, the degradation model can be referred to Section 3. For the controllimit policy of CBM within the finite horizon, the objective function is to minimize the expected maintenance cost rate for all degradation scenarios as follows:

$$
E C=\min _{d_{1}^{i} \in\left[d_{\min }, d_{\max }\right]} \sum_{i}^{N S} p_{i} Q\left(d_{1}^{i}, Z^{i}\right),
$$

where $p_{i}$ is the possibility of each degradation scenario. From (1), (2) and equations in control-limit policy, optimal PM threshold can be obtained for each selected scenario.

\section{Mathematical formulation for degradation forecasting model}

Since exponential degradation model is common for rotating machinery and bearing application [9, 19], we use this type of degradation model which is expressed as follows:

$$
W(t)=\phi+\theta \exp \left(\beta t+\varepsilon(t)-\frac{\sigma^{2}}{2} t\right),
$$

where $\phi$ is a known nonnegative constant, and $\theta$ is a lognormal random variable, $\beta$ is normal random variable, with mean $\mu_{1}$ and variance $\sigma_{1}^{2}$ and $\varepsilon(t)$ is the error term which follows a Brownian with mean 0 and variance $\sigma^{2}$. For simplification, the logarithm of degradation signal $l(t)$ is formed as:

$$
l(t)=\ln (W(t)-\phi)=\theta^{\prime}+\beta^{\prime} t+\varepsilon(t),
$$

where $\theta^{\prime}$ and $\beta^{\prime}$ are independent and follow normal distribution with mean $\mu_{0}$ and variance $\sigma_{0}^{2}$, and with mean $\mu_{1}^{\prime}$ and variance $\sigma_{1}^{2}$, respectively. Therefore, $\theta^{\prime}$ and $\beta^{\prime}$ can be expressed by $\theta^{\prime} \sim \mathrm{N}\left(\mu_{0}, \sigma_{0}^{2}\right)$ and $\beta^{\prime} \sim \mathrm{N}\left(\mu_{1}^{\prime}, \sigma_{1}^{2}\right)$, respectively. And $\mu_{1}^{\prime}=\mu_{1}-\sigma^{2} / 2, \beta^{\prime}=\beta-\sigma^{2} / 2, \phi$ is set to be 0.

PROPOSITION 1.The predictive distribution of the random variable $L$ of stochastic degradation is normal with mean and variance [19]:

$$
\begin{aligned}
& \mu\left(k, l_{k}\right)=l_{k}+\mu_{\beta}^{\prime}\left(k, l_{k}\right) \Delta t, \\
& \sigma^{2}(k)=\sigma_{\beta}^{\prime 2}(k) \Delta t^{2}+\sigma^{2} \Delta t .
\end{aligned}
$$

PROPOSITION 2. The posterior distribution of $\beta^{\prime}$ , is normal with the following parameters [19]:

$$
\begin{gathered}
\mu_{\beta}^{\prime}\left(k, l_{k}\right)=\frac{\left(\sigma_{1}^{2} l_{k}+\mu_{1}^{\prime} \sigma^{2}\right)\left(\sigma_{0}^{2}+\sigma^{2} \Delta t\right)-\sigma_{1}^{2}\left(l_{0} \sigma_{0}^{2}+\Delta t \mu_{0} \sigma^{2}\right)}{\left(\sigma_{0}^{2}+\sigma^{2} \Delta t\right)\left(\sigma_{1}^{2} k \Delta t+\sigma^{2}\right)-\Delta t \sigma_{0}^{2} \sigma_{1}^{2}}, \\
\sigma_{\beta}^{\prime}\left(k, l_{k}\right)=\frac{\sigma^{2} \sigma_{1}^{2}\left(\sigma_{0}^{2}+\sigma^{2} \Delta t\right)}{\left(\sigma_{0}^{2}+\sigma^{2} \Delta t\right)\left(\sigma_{1}^{2} k \Delta t+\sigma^{2}\right)-\Delta t \sigma_{0}^{2} \sigma_{1}^{2}} . \text { (8) time a } \\
\text { distrib }
\end{gathered}
$$

From Proposition 1 and 2, it can be seen that each time a signal is observed, it is used to update the predictive distribution of $L$. Therefore, it is a kind of non-stationary process for degradation, whose evolution is updated based 
on the monitoring condition for the system. For CBM, the optimal decision is made based on various forecasting degradation scenarios, since the degradation evolution process is stochastic within the planning horizon. Therefore, the initial scenarios can be generated through Proposition 1 and 2 by Monte Carlo simulation.

\section{Scenario reduction method based on output performance}

For CBM optimization combined with stochastic forecasting degradations, it is essential to make the scenario reduction to increase the calculation efficiency. Therefore, a kind of scenario reduction method based output performance is proposed, instead of isolating the scenario reduction from the problem. The procedures of this proposed method are shown in Fig. 2. It is based on the pattern recognition among the output performance (optimal PM threshold and the objective function value) and the forecasting degradation scenarios. Specific relationships can be derived from the analysis of the solutions for the CBM optimization with various degradation scenarios. The major procedure can be described by the following four steps.

Step 1. Monotonize the non-monotonic degradation processes.

Some degradation scenarios may be non-monotonic, and the non-monotonic degradation processes $W(T)$ are monotonized to be the monotonic degradations $Z(T)$ as follows:

$$
Z(T)=\max \{W(\tau): \tau \leq T\}
$$

Scenario generation

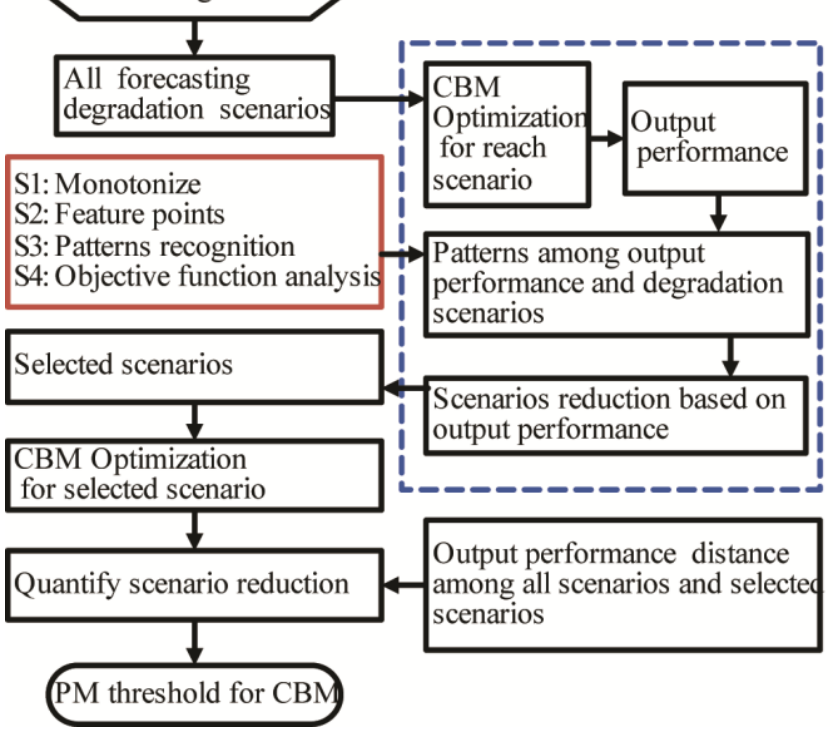

Fig. 2 Procedures of scenario reduction method based on output performance for CBM optimization

Step 2. Identify feature points from the monotonic degradations.

1) For degradation scenario $i$, check whether it will exceed the failure threshold within the planning horizon. If it does, the first point which is $\Delta Z$ higher than the failure threshold is set to be feature point, $Z_{1}$. Otherwise, there is no feature point $Z_{1}$ for scenario $i . Z_{1}$ can be formulated as follows:

$$
T_{1}=\min \left\{\tau: Z(\tau)+\Delta Z \geq Z_{\mathrm{F}}\right\}, Z_{1}=Z\left(T_{1}\right),
$$

where $\Delta Z$ is the unit of discrete degradation evolution, and $T_{1}$ is the corresponding time of $Z_{1}$. However, if there is no degradation over failure threshold, $T_{1}$ is set to be equal to $N T$.

2) For degradation scenario $i$, the first largest degradation point below the failure threshold $Z_{F}$ is set to be feature point $Z_{2}$, and alternatively, it can be formulated based on $T_{1}$ as follows:

$$
\begin{aligned}
& Z_{2}=\max \left\{Z(\tau): \tau \in\left[1, T_{1}-1\right]\right\}, \\
& T_{2}=\min \left\{\tau: Z(\tau)=Z_{2}\right\}, \\
& N_{2}=N\left(T_{2}\right)=\left\{q: T_{2} \in\left(\frac{N T}{q+1}, \frac{N T}{q}\right], q=1,2, \ldots\right\},
\end{aligned}
$$

where $T_{2}$ is the corresponding time for feature point $Z_{2}$. Meanwhile, $N\left(T_{2}\right)$ represents that the total number of PM actions needed within the finite planning horizon if the first maintenance action is made at time $T_{2}$. E.g. if $T_{2}=0.4 \mathrm{NT}$, so it satisfies $T_{2} \in(N T / 3, N T / 2]$, then $q=2$, and $N\left(T_{2}\right)=2$. That is, if the system is performed first maintenance at time $T_{2}=0.4 N T$, then the second maintenance will be performed at time $T_{2}=0.8 N T$ periodically and there will be two maintenance actions within the planning horizon. Moreover, it is assumed that the system is renewable after each maintenance action and it will follow the same degradation scenario as that before maintenance.

3) For degradation scenario $i$, search the first feature point $Z_{3}$ whose corresponding time $T_{3}$ satisfies that $T_{3}=\min \left\{t: N(t)=N\left(T_{2}\right)\right\}$. Alternatively, $T_{3}$ can be formulated based on $N\left(T_{2}\right)$ as follows:

$$
T_{3}=1+\frac{T}{1+N\left(T_{2}\right)}, Z_{3}=Z\left(T_{3}\right)
$$

where $Z\left(T_{3}\right)$ is the degradation evolution process for scenario $i$ during time period $T_{3}$. Meanwhile, $T_{3}$ is round number.

4) For degradation scenario $i$, find the last feature point $Z_{4}$ which satisfies that $T_{4}=\max \left\{t: N(t)=N\left(T_{2}\right)-1\right\}$. Alternatively, $Z_{4}$ can be formulated based on $T_{3}$ as follows:

$$
T_{4}=T_{3}-1, Z_{4}=Z\left(T_{4}\right)
$$

Step 3. Identify characteristic patterns among solutions and scenario feature points.

After identifying these four feature points from the degradation scenarios, there are four kinds of modes can be indentified with different relationships among these feature points, as shown in Fig. 3. 
For Mode 1, no degradations are over failure threshold, so there is no feature point $Z_{1}$. The lower and upper bound of the optimal threshold are, respectively, $d_{1 L}=Z_{2}+\Delta Z$ and $d_{1 U}=Z_{F}-\Delta Z$. Since no degradation points are over failure threshold, the number of PMs is zero within the planning horizon. For Mode 2, $Z_{1}$ exists and $Z_{2}=Z_{3}$. So, $d_{1 L}=Z\left(T_{2}-1\right)+\Delta Z$ and $d_{1 U}=Z_{2}$. For Mode $3, Z_{1}$ exists, $Z_{2}>Z_{3}$ and $Z_{3}=Z_{4}$. So, $d_{1 L}=Z_{3}+\Delta Z$ and $d_{1 U}=Z_{2}$. For Mode 4, $Z_{1}$ exists, $Z_{2}>Z_{3}$ and $Z_{3}>Z_{4}$. So, $d_{1 L}=Z_{4}+\Delta Z$ and $d_{1 U}=Z_{2}$.

For Mode 2, 3 and 4, the number of PM is $N\left(T_{2}\right)$ within the planning horizon.

Step 4. Objective function analysis.

From the relationship between modes and optimal solutions for different degradation scenarios, $N_{P M}$ can be determined by the specific modes of the degradation scenarios. The objective function is proportional to $N_{\mathrm{PM}}$, since the objective is to minimize the total maintenance cost. As shown in Fig. 3, for Mode 2, 3, 4, the total number of PMs is determined by the time when the first PM occurs. That is, it is determined by position of the feature point $Z_{2}$ in the degradation scenarios. For each degradation scenario, the optimal threshold is an interval-value data, instead of single-value data. Therefore, the scenarios share the same objective function value can be reduced to one representative scenario for the proposed method.

After the scenario reduction by the proposed method, optimal PM thresholds for CBM can be solved based on the selected degradation scenarios.
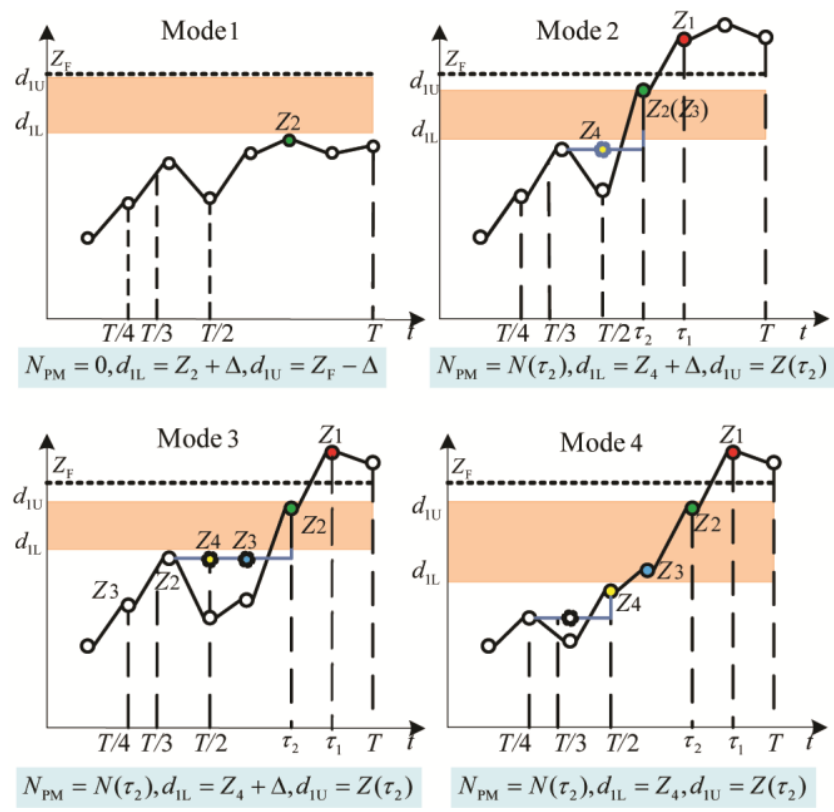

Fig. 3 Schematic diagram of feature points for degradation scenario integrating control-limit policy

\section{Case study}

\subsection{Original data}

For the application of proposed scenario reduction method based on output performance (short for PSR), we utilize the test data of vibration-based signals of the paper by Elwany, Gebraeel et al. [9]. According to the monitoring signals, exponential degradation model can be used to model the bearing degradation. The prior means, variances, and the variance parameter of the Brownian error terms are $\mu_{0}=-6.031, \mu_{1}^{\prime}=8.061 \times 10^{-3}, \sigma_{0}^{2}=0.346, \sigma_{1}^{2}=1.034 \times 10^{-5}$, $\sigma^{2}=0.0073$ respectively. The failure threshold is defined by the root mean square (RMS) of all over acceleration, and it is set to be $Z_{F}=0.025 V_{r m s}$ (root mean square volts) [9]. Cost data in this application including $c_{F}=\$ 120$ and $c_{P}=\$ 10$. The planning horizon is $N T=300 \mathrm{~min}$, and the time unit of degradations is set to be minute. For simplification, minute is the default time unit in section 5.2 and 5.3 unless otherwise stated.

\subsection{Scenario generation for degradation forecasting}

The models of randomness with their finite, discrete realizations are called scenario generators [20]. We generate $10^{3}$ degradation scenarios according to the exponential degradation model as mentioned in Section 3. Firstly, the initial signal value is initialized $\left(l_{0}=\log (0.001)=-6.9078\right)$. Secondly, the posterior distribution of $\beta^{\prime}$ can be obtained based on the signal value at time $t=0$. The forecasting signal value of $t=1$ can be stochastically chosen. Then the posterior distribution of $\beta^{\prime}$ of $t=2$ can be obtained accordingly, and so forth. Then the forecasting degradation state, $Z_{t}^{i}, i=1,2, \cdots, N T$, can be simulated accordingly.

\subsection{Results and discussions}

The initial $10^{3}$ degradation scenarios are reduced to 10 representative scenarios by PSR (Fig. 4), according to the objective function values. For each kind of degradation scenarios, which share the same value of objective function value, are replaced by average degradation scenario as one representative. Meanwhile, the possibility of the new representative scenario is added by that of the same kind of degradation scenarios. According to the proposed scenario reduction method, the probability of the reduced scenarios can be added to the representative scenario.

In this section, one of scenario reduction method based on input performance, fast forward selection ( short for FFS) [21], is chosen to make the comparison study to the proposed scenario reduction method, PSR. The initial degradation scenarios are reduced by FFS, and the relative possibility distance increases as the number of reduced scenarios decrease. The relative possibility distance is around 0.3 as the number of scenarios is reduced to 10 . The corresponding possibility and optimal solutions of each selected scenario are listed in Table 1 . Some of these 10 selected scenarios share the same number of PMs, instead of completely different. E.g. for scenario \#426, \#823, \#910 and \#1000, $N_{P M}=1$. Meanwhile, $N_{P M}$ for the rest of the selected scenarios are $0,2,3,5,6$, and 8 , respectively.

For the control-limit policy of CBM for singlecomponent system, the optimal threshold is interval-value data, instead of single-value data. Therefore, the output performance of CBM can be analysed based on the objective function value $(Q)$, optimal PM threshold $\left(d_{1}\right)$, the lower 
bound and upper bound for the optimal threshold ( $d_{1 L}$ and $\left.d_{1 U}\right)$.

Table 1

Ten selected scenarios by FFS

\begin{tabular}{|c|c|c|c|c|c|}
\hline Scenario & $\# 96$ & $\# 426$ & $\# 823$ & $\# 828$ & $\# 848$ \\
\hline$p_{i}$ & 0.001 & 0.094 & 0.211 & 0.001 & 0.079 \\
\hline$N_{P M}$ & 5 & 1 & 1 & 8 & 2 \\
\hline Scenario & $\# 866$ & $\# 910$ & $\# 923$ & $\# 990$ & $\# 1000$ \\
\hline$p_{i}$ & 0.017 & 0.249 & 0.033 & 0.286 & 0.029 \\
\hline$N_{P M}$ & 3 & 1 & 6 & 0 & 1 \\
\hline
\end{tabular}

Table 2

Output performance distance comparison

\begin{tabular}{|c|c|c|c|c|}
\hline $\begin{array}{c}\text { Compari- } \\
\text { son }\end{array}$ & $f$ or $N_{P M}$ & $d_{1}$ & $d_{1 U}$ & $d_{1 L}$ \\
\hline OPTI - FFS & 0.276 & 0.067 & 0.402 & 0.235 \\
\hline $\begin{array}{c}\text { OPTI - } \\
\text { PSR }\end{array}$ & $\mathbf{0 . 0 0 0}$ & $\mathbf{0 . 0 5 9}$ & $\mathbf{0 . 3 9 6}$ & 0.336 \\
\hline
\end{tabular}

The comparison results are shown in Fig. 5 and Table 2. Since objective function value is proportional to $N_{P M}$, the output performance of objective function value can be analysed by $N_{P M}$, as shown in Fig. 5, a. The probability distribution of $N_{P M}$ for selected scenarios (FFS, PSR) and initial scenarios (short for OPTI) are shown in Fig. 5, a. The probability distance is 0.276 between the probability distributions of $N_{P M}$ for selected scenarios by FFS and by OPTI. Meanwhile, the probability distance is 0.000 between the probability distributions of $N_{P M}$ for selected scenarios by PSR and by OPTI. Compared to FFS, the output performance, objective function value $Q$, of PSR is much closer to that of OPTI.

The probability distributions of $d_{1}$ for selected scenarios (FFS, PSR) and initial scenarios (OPTI) are shown in Fig. 5, b. The probability distance is 0.067 between the probability distributions of $d_{1}$ for selected scenarios by FFS and by OPTI. Meanwhile, the probability distance is 0.059 between the probability distributions of $d_{1}$ for selected scenarios by PSR and by OPTI. Compared to FFS, the output performance, $d_{1}$, of PSR is much closer to that of OPTI. The probability distributions of $d_{1 U}$ for selected scenarios (FFS, PSR) and initial scenarios are shown in Fig. 5, d. The probability distance is 0.402 between the probability distributions of $d_{1 U}$ for selected scenarios by FFS and by OPTI. Meanwhile, the probability distance is 0.396 between the probability distributions of $d_{1 U}$ for selected scenarios by PRS and by OPTI. Compared to FFS, the output performance distance, $d_{1 U}$, of PSR is much closer to that of OPTI.

However, the probability distributions of $d_{1 L}$ for selected scenarios and initial scenarios are shown in Fig. 5,. The probability distance is 0.235 between the probability distributions of $d_{1 L}$ for selected scenarios by FFS and by OPTI. Meanwhile, the probability distance is 0.336 between the probability distributions of $d_{1 L}$ for selected scenarios by PRS and by OPTI. Compared to FFS, the output performance distance, $d_{1 L}$, of PSR is a little bit greater to that of OPTI. It is the pay for the distance reduction of objective function value and $d_{1}$ by the proposed scenario reduction method, PSR.
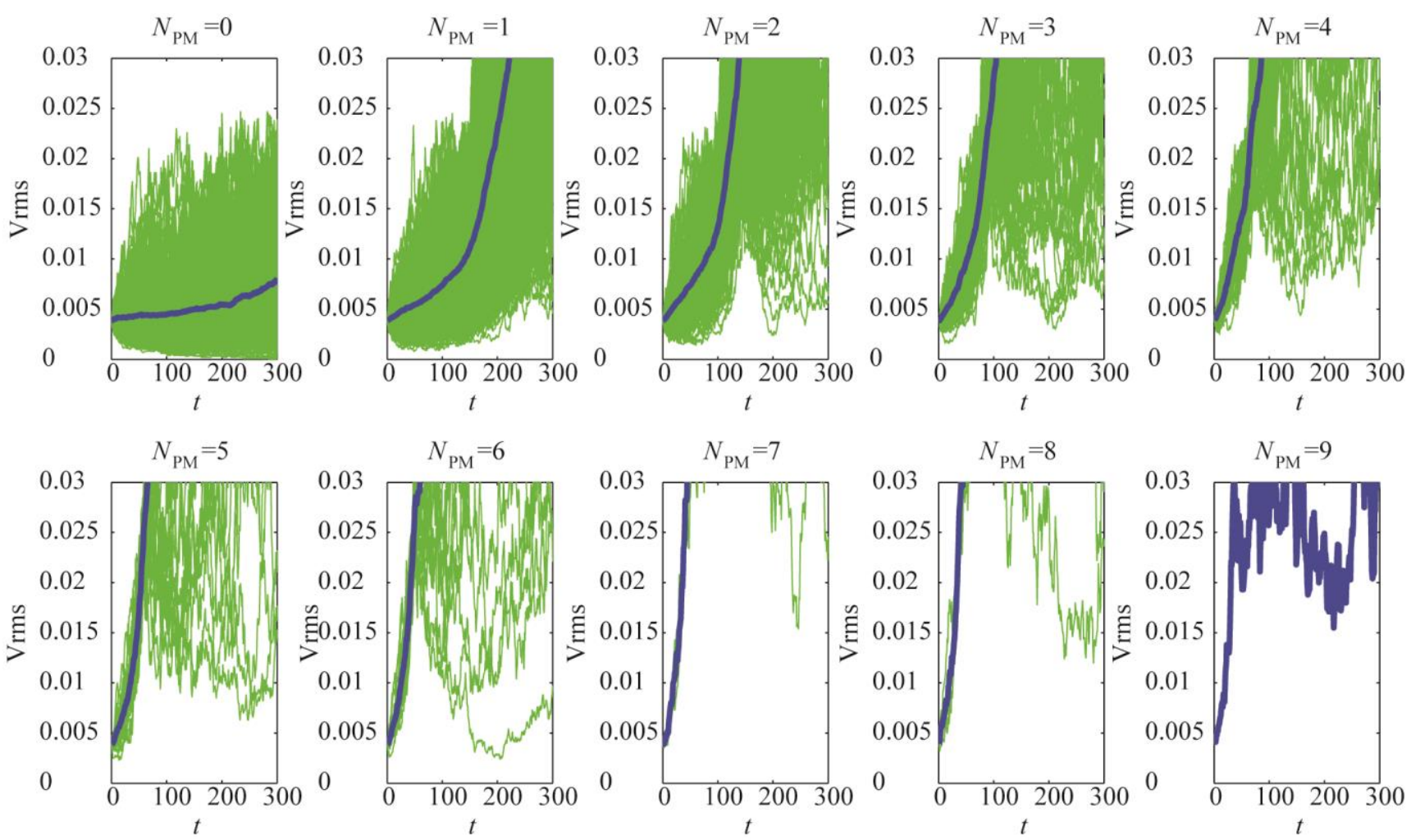

Fig. 4 Ten kinds of scenarios by PSR

From the comparison of the output performance for selected scenarios and initial scenarios, it shows that both the objection value $\left(Q\right.$ or $\left.N_{P M}\right)$ and optimal threshold $\left(d_{1}\right)$ distribution distance between selected scenarios by PSR and initial scenarios are much closer than that between selected scenarios by FFS and initial scenarios. The reason is that the proposed scenario reduction method can consider the role of 
the uncertainty in the CBM model. Moreover, it can capture the characteristics, which have influence on the output performance. Therefore, the scenarios with same kind of features can be reduced to one representative scenario.
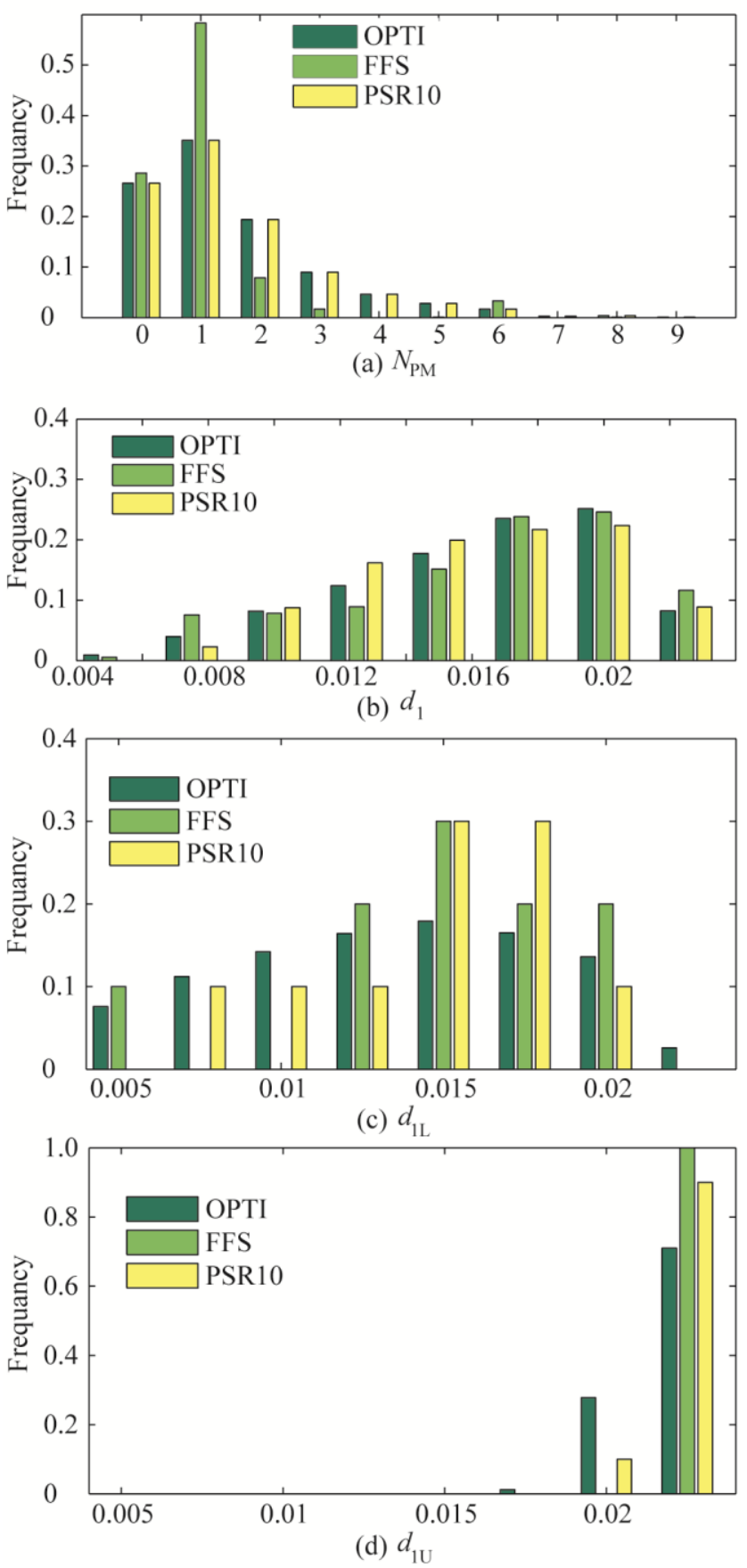

Fig. 5 Probability distributions of output performance ((a) number of PMs $N_{P M}$, (b) PM threshold $d_{1}$, (c) Lower bound of PM threshold $d_{1 L}$ and (d) Upper bound of PM threshold $d_{1 U}$ ) for scenarios by FFS, PSR and OPTI

\section{Conclusions}

To improve the efficiency of scenario reduction for CBM problem involving uncertainties, such as stochastic degradation, a kind of scenario reduction method based on the output performance is proposed. This scenario reduction method can utilize the influence of the scenarios on their output performance, instead of input performance of scenarios. Therefore, the proposed method can increase the efficiency of scenario reduction so as to reduce computational complexity for CBM in finite horizon.

Further extension of this work will focus on investigating the feature modes for CBM policy of multi-component system that based on the proposed scenario reduction method. In addition, we assume that the state after maintenance is as good as mew. It is also possible to consider the case that the maintenance effect is partial repair or stochastic.

\section{Acknowledgments}

This work is supported by National Natural Science Foundation of China (Grant Nos. 71501148, 51275366, 51505349). The research work is also supported by National Science and Technology Major Project of China (Grant No. 2016ZX05038-002-LH002).

\section{References}

1. Jardine, A.K.S.; Lin, D.M.; Banjevic, D. 2006. A review on machinery diagnostics and prognostics implementing condition-based maintenance, Mechanical Systems and Signal Processing 20(7): 1483-1510. http://dx.doi.org/10.1016/j.ymssp.2005.09.012.

2. Lee, J.;Wu, F.; Zhao, W.; Ghaffari, M.; Liao, L.; Siegel, D. 2014. Prognostics and health management design for rotary machinery systems-Reviews, methodology and applications, Mechanical Systems and Signal Processing 42(1-2): 314-334.

http://dx.doi.org/10.1016/j.ymssp.2013.06.004.

3. Gorjian, N.; Ma, L.; Mittinty, M.; Yarlagadda, P.; Sun, Y. 2009. A review on degradation models in reliability analysis. In Proceedings of the 4th World Congress on Engineering Asset Management. Athens. http://dx.doi.org/10.1007/978-0-85729-320-6_42.

4. Li, C.J.; Lee, H. 2005. Gear fatigue crack prognosis using embedded model, gear dynamic model and fracture mechanics, Mechanical systems and signal processing 19(4): 836-846.

http://dx.doi.org/10.1016/j.ymssp.2004.06.007.

5. Sun, J.; Zuo, H.; Wang, W.; Pecht, M.G. 2014. Prognostics uncertainty reduction by fusing on-line monitoring data based on a state-space-based degradation model, Mechanical Systems and Signal Processing 45(2): 396-407.

http://dx.doi.org/10.1016/j.ymssp.2013.08.022.

6. Benmounah, A. 2014. Optimization of maintenance intervals preventive and repair of gas turbines. Case of Algerian gas pipelines, Mechanika 20(3): 305-309. http://dx.doi.org/10.5755/j01.mech.20.3.7394.

7. Dragomir, O. E.; Gouriveau, R.; Dragomir, F.; Minca, E.; Zerhouni, N. 2009. Review of prognostic problem in condition-based maintenance, European Control Conference, ECC'09., Budapest : Hungary. https://hal.archives-ouvertes.fr/hal-00418761.

8. Nakagawa, T.; Mizutani, S. 2009. A summary of maintenance policies for a finite interval, Reliability Engineering \& System Safety 94(1): 89-96. http://dx.doi.org/10.1016/j.ress.2007.04.004.

9. Elwany, A.H.; Gebraeel, N.Z.; Maillart, L.M. 2011. Structured Replacement Policies for Components with 
Complex Degradation Processes and Dedicated Sensors, Operations Research 59(3): 684-695.

http://dx.doi.org/10.1287/opre.1110.0912.

10. Alrabghi, A.; Tiwari, A. 2015. State of the art in simulation-based optimisation for maintenance systems, Computers \& Industrial Engineering 82: 167-182. http://dx.doi.org/10.1016/j.cie.2014.12.022.

11. Li, Z.; Floudas, C.A. 2014. Optimal scenario reduction framework based on distance of uncertainty distribution and output performance: I. Single reduction via mixed integer linear optimization, Computers \& Chemical Engineering 70(0): 50-66.

http://dx.doi.org/10.1016/j.compchemeng.2014.03.019.

12. Feng, Y.; Ryan, S.M. 2013. Scenario construction and reduction applied to stochastic power generation expansion planning, Computers \& Operations Research 40(1): 9-23. http://dx.doi.org/10.1016/j.cor.2012.05.005.

13. De Oliveira, W.L.; Sagastizábal, C.; Penna, D.D.J.; Maceira, M.E.P.; Damázio, J.M. 2010. Optimal scenario tree reduction for stochastic streamflows in power generation planning problems, Optimisation Methods \& Software 25(6): 917-936.

http://dx.doi.org/10.1080/10556780903420135.

14. Dupačová, J.; Gröwe-Kuska, N.; Römisch, W. 2003. Scenario reduction in stochastic programming: an approach using probability metrics, Mathematical programming 95(3): 493-511. http://dx.doi.org/10.1007/s10107-002-0331-0.

15. Heitsch, H.; Römisch, W. 2009. Scenario tree modeling for multistage stochastic programs, Mathematical Programming 118(2): 371-406. http://dx.doi.org/10.1007/s10107-007-0197-2.

16. Sumaili, J.; Keko, H.; Miranda, V.; Zhou, Z.; Botterud, A.; Wang, J. 2011. Finding representative wind power scenarios and their probabilities for stochastic models. In Intelligent System Application to Power Systems (ISAP), 2011 16th International Conference on. IEEE. http://dx.doi.org/10.1109/ISAP.2011.6082195.

17. Zhao, X.; Fouladirad, M.; Bérenguer, C.; Bordes, L. 2010. Condition-based inspection/replacement policies for non-monotone deteriorating systems with environmental covariates, Reliability Engineering \& System Safety 95(8): 921-934. http://dx.doi.org/10.1016/j.ress.2010.04.005.

18. Tian, Z.; Liao, H. 2011. Condition based maintenance optimization for multi-component systems using proportional hazards model, Reliability Engineering \& System Safety 96(5): 581-589.

http://dx.doi.org/10.1016/j.ress.2010.12.023.

19. Gebraeel, N.Z.; Lawley, M.A.; Li, R.; Ryan, J.K. 2005. Residual-life distributions from component degradation signals: A Bayesian approach, IIE Transactions 37(6): 543-557.

http://dx.doi.org/10.1080/07408170590929018.

20. Di Domenica, N.; Mitra, G.; Valente, P.; Birbilis, G. 2007. Stochastic programming and scenario generation within a simulation framework: an information systems perspective, Decision Support Systems 42(4): 21972218 . http://dx.doi.org/10.1016/j.dss.2006.06.013.

21. Growe-Kuska, N.;Heitsch, H.; Romisch, W. 2003. Scenario reduction and scenario tree construction for power management problems. In 2003 IEEE Bologna Power Tech Conference Proceedings. http://dx.doi.org/10.1109/PTC.2003.1304379.

Xinbo Qian, Qiuhua Tang

\section{SCENARIO REDUCTION METHOD BASED ON OUTPUT PERFORMANCE FOR CONDITION-BASED MAINTENANCE OPTIMIZATION}

S u m m a r y

Condition-based maintenance (CBM) has been widely applied to maintenance policy. Due to the stochastic forecasting degradation, scenario reduction method has been developed to improve the efficiency of CBM. However, most existing scenario reduction methods focus mainly on the input performance of the forecasting degradation without considering the significant output performance characteristic based on the model.

In order to warrant the CBM optimization precision while reducing stochastic degradation scenarios efficiently, a new scenario reduction method is formulated that the scenarios with same objective function values can be reduced to one representative scenario. As a result, the reduced scenarios by the proposed method can maintain the probability distributions of objective values, while keeping optimal thresholds close to that of initial scenarios. Finally, the method is applied to select the representative degradation scenarios for CBM optimization model by utilizing vibration-based degradation signals from a rotating machinery application. Compared to the traditional scenario reduction method, the proposed method further improves accuracy and reduction efficiency of CBM optimization.

Keywords: condition-based maintenance, degradation, scenario reduction, output performance, probability, preventive maintenance, optimization.

Received July 01, 2016

Accepted October 13, 2017 